\title{
Apport de la tomodensitométrie et de la reconstruction tridimensionnelle dans le traitement de l'ankylose : une thérapeutique originale sur 5 cas
}

\author{
Paris $\mathrm{M}^{1}$, Trunde $\mathrm{F}^{1}$, Bossard $\mathrm{D}^{2}$, Coudert $\mathrm{JL}^{1}$ \\ ${ }^{1}$ Service de Consultations et de Traitements dentaires, Hospices Civils, Lyon, France \\ ${ }^{2}$ Clinique Saint Jean, Lyon, France \\ parmaceci@hotmail.com
}

L'ankylose des dents permanentes est une pathologie peu fréquente due à la fusion anormale du cément (voire de la dentine) de la dent atteinte à l'os alvéolaire sans interposition de tissu conjonctif. Le phénomène d'ankylose et son traitement sont plus particulièrement analysés dans cette série clinique.

Classiquement, lorsqu'une dent fait l'objet d'un diagnostic d'ankylose, les solutions thérapeutiques envisageables sont peu nombreuses. Lorsque la dent est déjà en place sur l'arcade il existe deux alternatives thérapeutiques. La dent peut être soit extraite soit maintenue sur l'arcade jusqu'à ankylose complète de la racine. Dans le premier cas, une solution implantaire ou orthodontique est immédiatement envisageable, dans le second cas, elle est différée. Lorsque la dent est incluse et que le diagnostic d'ankylose est confirmé, les seules solutions thérapeutiques envisageables sont l'extraction de la dent ou bien l'abstention. Nous proposons une nouvelle alternative thérapeutique à l'ankylose pour une dent incluse.

Sur un ensemble de patients, adressés par des orthodontistes, présentant des dents incluses et retenues d'étiologie inconnue ou des dents avec un échec de traction, 15 ont fait l'objet d'un diagnostic d'ankylose ( $7 \mathrm{du}$ groupe incisivo-canin, 3 prémolaires, 5 molaires) grâce à un examen tomodensitométrique. Une analyse d'image ainsi qu'une reconstitution tridimensionnelle sont réalisées grâce au logiciel MVS (Hospices civils de Lyon) à l'aide d'un algorithme de segmentation d'image basé sur le principe de la croissance de région. Elle permet de préciser le volume radiculaire concerné par l'ankylose et de visualiser sa localisation précise sur la dent. Sur ces 15 dents incluses, 5 dents dont les conditions anatomiques le permettaient ont fait l'objet d'une thérapeutique originale.

Le protocole opératoire est relativement simple et facile à mettre en œuvre. La dent ankylosée est précisément localisée à l'aide de la reconstitution tridimensionnelle ainsi que la zone ankylosée sur la dent. Puis, la dent est extraite, la zone d'ankylose est supprimée à l'aide d'une fraise boule diamantée sous irrigation et remplacée par un ciment verre ionomère à prise rapide. Enfin la dent est replacée dans son alvéole et un bracket collé sur la dent afin de commencer la traction de la dent.

Toutes les dents ont pu être tractées sans difficulté sur l'arcade. Une observation sur une durée plus longue permettra d'observer si le phénomène d'ankylose se poursuit sur la dent après son mise en place sur l'arcade ou bien si la dent se comporte ensuite comme une dent sans ankylose. 